\title{
Ophthalmologic Psychophysical Tests Support OCT Findings in Mild Alzheimer's Disease
}

\author{
Elena Salobrar-Garcia, ${ }^{1}$ Rosa de Hoz, ${ }^{1,2}$ Blanca Rojas, ${ }^{1,3}$ Ana I. Ramirez, ${ }^{1,2}$ Juan J. Salazar, ${ }^{1,2}$ \\ Raquel Yubero, ${ }^{4}$ Pedro Gil, ${ }^{3,4}$ Alberto Triviño, ${ }^{1,3}$ and José M. Ramirez ${ }^{1,3}$ \\ ${ }^{1}$ Instituto de Investigaciones Oftalmológicas Ramón Castroviejo, Universidad Complutense de Madrid, Madrid, Spain \\ ${ }^{2}$ Facultad de Óptica, Universidad Complutense de Madrid, Madrid, Spain \\ ${ }^{3}$ Facultad de Medicina, Universidad Complutense de Madrid, Madrid, Spain \\ ${ }^{4}$ Hospital Clínico San Carlos, Madrid, Spain \\ Correspondence should be addressed to José M. Ramirez; ramirezs@med.ucm.es
}

Received 19 February 2015; Accepted 20 May 2015

Academic Editor: Hermann Mucke

Copyright (C) 2015 Elena Salobrar-Garcia et al. This is an open access article distributed under the Creative Commons Attribution License, which permits unrestricted use, distribution, and reproduction in any medium, provided the original work is properly cited.

\begin{abstract}
Purpose. To analyze in mild Alzheimer's disease (MAD) patients, GDS-4 (Reisberg Scale), whether or not some psychophysical tests (PTs) support OCT macular findings in the same group of MAD patients reported previously. Methods. Twenty-three MAD patients and 28 age-matched control subjects with mean Mini Mental State Examination of 23.3 and 28.2, respectively, with no ocular disease or systemic disorders affecting vision were included. Best-corrected visual acuity (VA), contrast sensitivity (CS) (3, 6, 12, and $18 \mathrm{cpds}$ ), color perception (CP), and perception digital test (PDT) were tested in one eye of each patient. Results. In comparison with the controls, MAD patients presented (i) a significant decrease in VA, PDT, and CS for all spatial frequencies analyzed, especially the higher ones, and (ii) a significant increase in unspecific errors on the blue axis $(P<0.05$ in all instances). In MAD patients, a wide aROC curve was plotted in all PTs. Conclusions. In MAD, CS, VA, and the tritan axis in CP were impaired. The PTs with the greatest predictive value are the higher spatial frequencies in CS and tritan unspecific errors in CP. PT abnormalities are consistent with the structural findings reported in the same MAD patients using OCT.
\end{abstract}

\section{Introduction}

Alzheimer's disease (AD) is a neurodegenerative disorder of the central nervous system characterized by cortical atrophy most pronounced in the medial temporal and posterior temporoparietal regions [1]. Disturbances of short-term memory, judgment, and emotion are characteristic of the disease.

As an extension of the central nervous system, the retina displays similarities to the brain in terms of anatomy, functionality, response to insult, and immunology [2-4]. Furthermore, the retina presents manifestations of major neurodegenerative diseases, and it has been argued that several ocular diseases should be viewed as forms of neurodegenerative disorders [2]. This unique property makes the retina a valuable tool for in vivo visualization and study of retinal changes.
The first histopathologic evidence of postmortem retinal changes from $\mathrm{AD}$ patients was reported by Hinton et al., who found a significant axonal degeneration in the optic head nerve [5]. Further studies have demonstrated that a decrease in retinal ganglion cell numbers was associated with an array of intracellular injuries [6]. The analysis on the distribution of neuronal loss in the retina by Blanks et al. showed that the most pronounced areas of cell loss in the ganglion cell layer (GCL) were the superior and inferior retinal quadrants [7] and there was an extensive loss of RGC in the temporal foveal region [8]. These findings have been corroborated using the Optical Coherence Tomography (OCT), demonstrating the decrease of the RNFL and the macular thickness [9-16], even in early stages of mild cognitive impairment $[12,17-19]$.

Currently it is thought that RGC loss in AD might result from amyloid pathology in the retina. Both amyloid-beta 
plaques and oligomers have been reported in the human retina [4, 20-25]. Therefore, amyloid accumulation in the retina of patients with $\mathrm{AD}$ may result in the degeneration of RGCs in parallel with amyloid-beta-related neurodegeneration in the brain [25]. However, retinal findings in AD could be also due to retrograde degeneration of RGCs secondary to $\mathrm{AD}$ pathology in the central visual pathways, given that AD patients show degeneration of the primary and secondary visual cortex [26].

In addition to the anatomical findings in $\mathrm{AD}$ patients, this disease can exert an impact on most aspects of visual processing, such as visual field abnormalities [27-29], color perception deficits [30-32], pattern electroretinogram changes $[11,33,34]$, and reduced contrast sensitivity (CS) [35-38].

The $M$ visual pathway appears to be particularly vulnerable to degeneration in AD [26]. Thus, degeneration of RGCs and/or visual pathways in the brain may underlie the functional deficits in CS seen in patients with AD [29]. CS function in humans diminishes with changes in the retina, the optic nerve, and lesions of the occipital, temporal, and/or parietal cortices [39].

Psychophysical investigations of CS in AD patients have rendered results consistent with the neuropathological evidence. However, studies of CS in patients with AD have yielded variable results. Some studies have reported no ADrelated deficits in spatial CS $[40,41]$, while others have found deficits at all spatial frequencies tested [42].

OCT macular studies in AD by our group and others have recently reported that mild $\mathrm{AD}(\mathrm{MAD})$ patients with a high mean score $(23.3 \pm 3.1)$ on the Mini Mental State Examination (MMSE) had significantly reduced macular nerve fiber layer thickness with or without significant peripapillary involvement $[9,43,44]$. In the present study, to analyze whether these anatomical features coexist with alterations in the visual functional state in MAD patients, we include the same patients from the previous work [9]. To examine the visual pathway in this homogeneous group (in terms of disease stage) of MAD, we performed different psychophysical tests, specifically visual acuity (VA), CS, color perception, and perception digital test (PDT). The results are compared with those of an age-matched control group in order to (i) improve our understanding on how the visual pathway works in MAD and (ii) determine whether or not these tests support early macular findings on OCT in MAD.

\section{Materials and Methods}

2.1. Subjects. To select patients, we reviewed the Database of the Memory Unit of the Hospital Clínico San Carlos in Madrid (Spain), consisting of a total of 2635 patients. First, we excluded the patients with a Global Deterioration Scale (GDS) over 4 [45] and then those with a mood or psychiatric disorder. Next, we took into account 87 patients with MAD. These patients, according to the National Institute of Neurological and Communicative Disorders and StrokeAlzheimer's Disease and Related Disorders Association and the Diagnostic and Statistical Manual of Mental Disorders IV, had mild cognitive impairment according to the Clinical Dementia Rating Scale. Then ophthalmic medical records of these patients were reviewed, excluding those patients who were previously diagnosed with an ophthalmic condition. After this analysis, 29 patients with AD had all the requirements to participate in the study (GDS over 4 and freedom of ocular disease and systemic disorders affecting vision in their medical record). Of the 29 MAD patients and 37 age-matched control subjects selected (normal MMSE scores), 6 MAD patients and 9 age-matched control subjects were subsequently excluded due to posterior pole pathology including macular degeneration, drusen, suspicion of glaucoma, glaucoma, epiretinal membrane, or cataract that prevented ocular examination. Because of this selection, 23 patients with MAD and 28 age-matched control subjects were considered for the study. Informed consent was obtained from both groups. The research followed the tenets of the Declaration of Helsinki, and the protocol was approved by the local ethics committee.

2.2. Methods. The clinical evaluation of our MAD included a review of the medical records, a caregiver interview, physical and neurological examinations, a psychometric test, neuroimaging techniques, and routine laboratory testing for dementia.

For the ophthalmological part of the study only one eye of each patient was analyzed. All participants met the following inclusion criteria: being free of ocular disease, AREDS Clinical Lens Standards $<2$, retinal drusen and systemic disorders affecting vision, having a best-corrected VA of 20/40, having a \pm 5 spherocylindrical refractive error, and having intraocular pressure of less than $20 \mathrm{mmHg}$. For screening, all AD patients and control subjects underwent a complete ophthalmologic examination, including assessment of VA, refraction, anterior segment biomicroscopy, applanation tonometry (Perkins MKII tonometer, Haag Streit, Reliance Medical, Switzerland), CS test CSV-1000E (VectorVision, Greenville, OH, USA), Roth 28-hue color test (Luneau, Paris, France), PDT [46], and dilated fundus examination. In the dilated fundus examination, no differences were found between MAD patients and age-matched control subjects. These tests were selected considering that in this developmental stage of the disease the results were not influenced by the patient's cognitive impairment.

2.3. Visual Acuity. Monocular best-corrected distant VA was determined using a standard clinical Snellen eye chart. The correction was based on the subjective refraction of the subject. The patients started to read each row from the top of the Snellen eye chart and proceeded toward the bottom. This was ended when the hit rate was less than five of eight (an approximation to 56.25\%, the steepest point of the psychometric acuity function).

2.4. Contrast Sensitivity Function. The CS test was performed under the same conditions for all the patients and with the CSV-1000E system (VectorVision, Greenville, OH, USA) and in the presence of best-corrected VA for far vision. The CSV-1000E test provides a fluorescent luminance source that retroilluminates a translucent chart and is able to monitor 
and autocalibrate the light level to $85 \mathrm{~cd} / \mathrm{m}^{2}$. The CSV$1000 \mathrm{E}$ was performed at 98.5 inches as recommended by the manufacturer. The nonselected eye was occluded for each measurement. The translucent chart presents four spatial frequencies: 3, 6, 12, and 18 cyc/deg. Each spatial frequency was presented on a separate row of the test. Each row presented 17 circular patches 1.5 inches in diameter. The first patch in the row had a very high contrast grating (sample patch) on the far-left side of the row. The remaining 16 patches appeared in 8 columns presented across the row. In each column, one patch presented a grating while the other patch remained blank. The patches that presented gratings decreased in contrast from left to right across the row. The patient was directed to observe the first sample patch and to look for the grating pattern in each column. While reading across the row, the patient indicated whether the grating appeared in the top patch or the bottom patch of each column. If the grating was not visible in either patch, the patient was to report that both were blank. The patient was encouraged to guess whether a grating was at least partially visible as the threshold was approached. However, the patient was cautioned that if no gratings were visible, then the response should be "both blank." The contrast level of the last correct response was recorded as the threshold.

2.5. Color Perception. To administer a color-vision test that did not require a naming response, we used Roth 28 -hue color test (Luneau, Paris), a quick and easy color arrangement test first described by Roth [47]. Color perception was assessed in the presence of best-corrected VA for near vision. The test uses the equivalent of every third color cap from the Farnsworth-Munsell 100 (F-M 100) as an abbreviated version. Subjects were instructed to select the cap most similar to the reference cap, then the cap most similar to the previously chosen one, and so on and to place them in sequential order until all 27 caps were arranged in a circular sequence. Test instructions were repeated by the examiner during test when necessary. The time to perform the test was not restricted and the subject was allowed to make corrections. The results were recorded on diagrams provided by the manufacturer which depict the direction of axes corresponding to several types of color-vision defects. Errors classified the observer as protanomalous, deuteranomalous, or tritanomalous (red-, green-, or blue-deficient, resp.). Following the manufacturer's manual [48] blue axis errors were considered when caps 43 to 64 were malpositioned. In this way, the tritan errors were quantified.

2.6. Perception Digital Test (PDT). The PDT, developed in 2007 [46], is an easy, fast, and sensitive method for evaluating disorders of visual perception in MAD patients. The aim of the test is to assess the visual recognition of familiar situations, masked by geometric special effects that hinder perception. The test includes 15 sheets. Each sheet shows the same picture at different positions in space. The pictures are distorted by the choice of special effects: geometric effect (tile) and effect of the frame 24/48 of MGI Photo Suite III program. The test includes five photographs of landscapes, six of common objects, two of people, one of an animal, and
TABLE 1: Demographic and clinical data of the study groups.

\begin{tabular}{lccc}
\hline & $\begin{array}{c}\text { AD } \\
(n=23)\end{array}$ & $\begin{array}{c}\text { Control } \\
(n=28)\end{array}$ & $P$ value \\
\hline Age $^{*}$ & $79.3 \pm 4.6$ & $72.3 \pm 5.1$ & 0.274 \\
Gender & & & 0.614 \\
$\quad$ Men & 9 & 9 & \\
Female & 14 & 19 & \\
Race & Caucasian & Caucasian & \\
& $23.3 \pm 3.1$ & $28.2 \pm 1.9$ & $0.001^{\dagger \dagger}$ \\
MMSE $^{*}$ & Range $(17-29)$ & Range $(25-31)$ & \\
Educational level $^{*}$ & $1.43 \pm 0.78$ & $1.43 \pm 0.79$ & 0.950 \\
\hline
\end{tabular}

${ }^{*}$ Mean value $\pm \mathrm{SD} ;{ }^{\dagger \dagger} P<0.01$ Mann-Whitney $U$ test $(\mathrm{AD}$ : Alzheimer's disease; MMSE: Mini Mental State Examination; SD: standard deviation).

one of a letter. The patient had to identify the picture that was properly oriented in space.

2.7. Statistical Analysis. Data for the statistical analysis were introduced and processed in SPSS 19.0 (SPSS Inc.@), Inc., Chicago, IL, USA). The data are reported as mean values \pm $\mathrm{SD}$. The differences between MAD and control eyes were analyzed using the Mann-Whitney test. Sensitivity at $90 \%$ specificity and area under the receiver operator characteristic (aROC) analysis for discriminating between healthy and MAD patients were calculated for all the psychophysical tests analyzed. The association between the tests and MMSE was evaluated by Pearson's correlation coefficient. A $P$ value of $<0.05$ was considered statistically significant.

\section{Results}

Demographic and clinical data for the MAD patients and control group are shown in Table 1 . There were no statistically significant differences in age, gender, or educational level between the study groups. The MMSE scores in MAD patients were significantly decreased in comparison with agematched control subjects (Table 1). All MAD patients had MMSE values higher than 17.

3.1. Visual Acuity. The mean VA in MAD patients significantly decreased in comparison with the age-matched control group (Table 2; Figure 1(a)). VA showed a wide aROC (Table 3; Figure 2(a)). A positive and statistically significant linear association was found between VA and MMSE score (Table 4).

3.2. Contrast Sensitivity. All analyses were conducted with $\log$ CS values. $30.43 \%$ of the MAD patients $(n=7)$ were not able to report the orientation of the 18-cpd grating at the highest contrast level. Three of these 7 MAD patients were also unable to detect the 12-cpd grating at any contrast value. The analysis of CS of the MAD patients revealed a statistically significant reduction at all spatial frequencies tested $(3,6$, 12 , and $18 \mathrm{cpds})$ in comparison with age-matched control subjects (Table 2; Figure 1(b)). In addition, it was found that the higher the spatial frequency, the greater the loss of CS 
TABLE 2: Mean data and $P$ value of the psychophysical tests.

\begin{tabular}{|c|c|c|c|c|}
\hline Test & AD group & Control group & $\%$ difference & $P$ value \\
\hline Visual acuity $(\mathrm{dec})^{*}$ & $0.78 \pm 0.19$ & $0.95 \pm 0.06$ & -17.89 & $0.001^{\dagger \dagger}$ \\
\hline \multicolumn{5}{|l|}{ Contrast sensitivity $^{*}$} \\
\hline $3 \mathrm{cpds}$ & $1.39 \pm 0.27$ & $1.62 \pm 0.19$ & -14.61 & $0.001^{\dagger \dagger}$ \\
\hline $6 \mathrm{cpds}$ & $1.58 \pm 0.24$ & $1.88 \pm 0.22$ & -16.03 & $0.001^{\dagger \dagger}$ \\
\hline $12 \mathrm{cpds}$ & $1.12 \pm 0.33$ & $1.46 \pm 0.20$ & -23.05 & $0.001^{\dagger \dagger}$ \\
\hline $18 \mathrm{cpds}$ & $0.61 \pm 0.37$ & $1.11 \pm 0.27$ & -44.57 & $0.001^{\dagger \dagger}$ \\
\hline \multicolumn{5}{|l|}{ Rue 28-hue* } \\
\hline Number of errors & $7.41 \pm 4.69$ & $5.95 \pm 4.06$ & 24.54 & 0.18 \\
\hline Tritan unspecific errors & $4.35 \pm 2.40$ & $2.52 \pm 2.00$ & 72.62 & $0.009^{\dagger \dagger}$ \\
\hline \multicolumn{5}{|l|}{ PDT (sheet) ${ }^{*}$} \\
\hline Successful number of sheets & $11.74 \pm 2.39$ & $13.79 \pm 1.47$ & -14.87 & $0.01^{\dagger}$ \\
\hline
\end{tabular}

${ }^{*}$ Mean value $\pm \mathrm{SD} ;{ }^{\dagger} \mathrm{P}<0.05,{ }^{\dagger \dagger} \mathrm{P}<0.01 \mathrm{Mann}-$ Whitney $U$ test (AD: Alzheimer’s disease; cpds: cycles per degree; PDT: perception digital test).

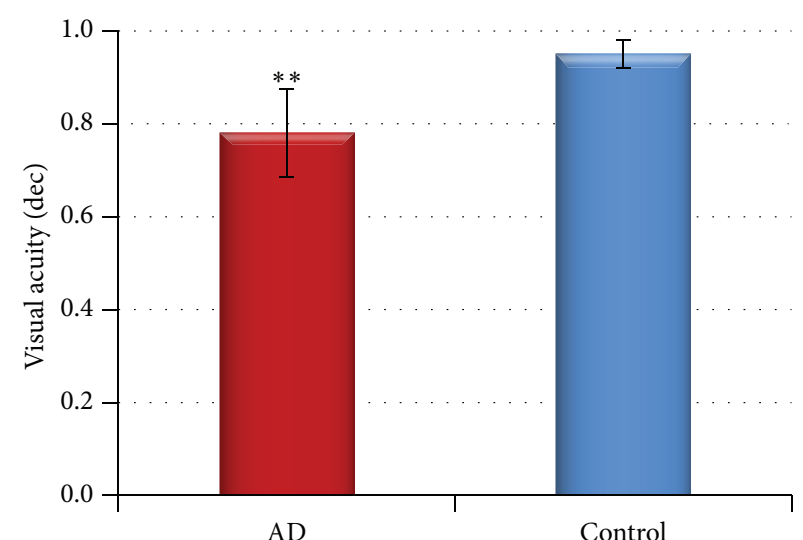

$\mathrm{AD}$

Control

(a) Visual acuity

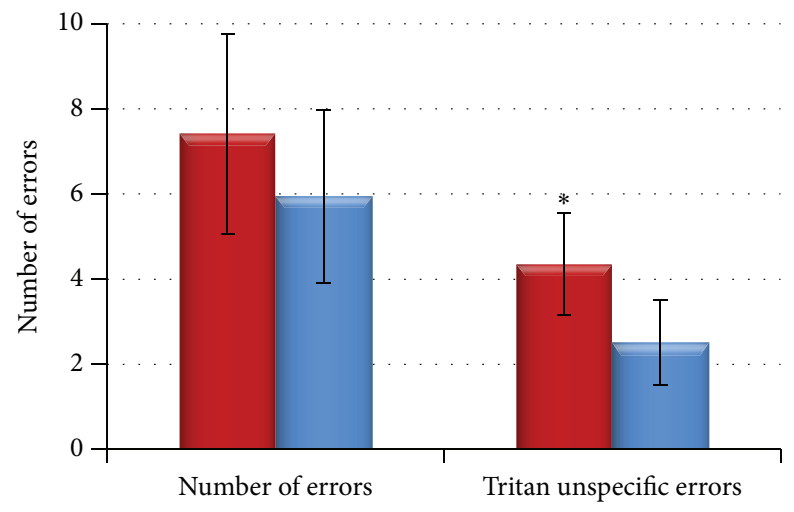

$\mathrm{AD}$

Control

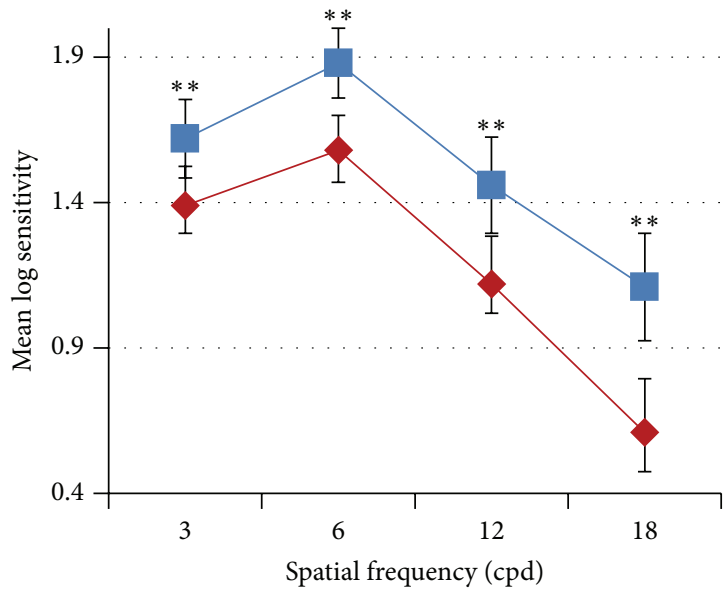

$\mathrm{AD}$

Control

(b) Contrast sensitivity

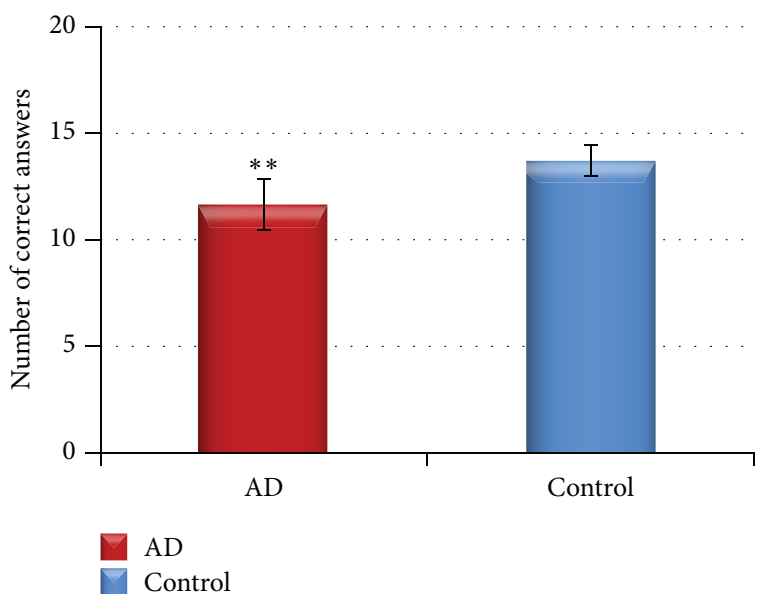

(d) PDT

Figure 1: Mean data of the psychophysical tests. (a) Visual acuity, (b) contrast sensitivity, (c) desaturated Rue 28-hue color test, and (d) perception digital test. Each bar represents the mean \pm SD. ${ }^{*} P<0.05$ versus control. ${ }^{* *} P<0.01$ versus control. Mann-Whitney $U$ test. 
TABLE 3: aROC analysis of psychophysical tests in mild Alzheimer's disease.

\begin{tabular}{|c|c|c|c|}
\hline Psychophysical test & aROC & SD & $P$ value \\
\hline Visual Acuity & 0.771 & 0.072 & $0.001^{\dagger \dagger}$ \\
\hline \multicolumn{4}{|l|}{ Contrast sensitivity } \\
\hline $3 \mathrm{cpds}$ & 0.755 & 0.072 & $0.002^{\dagger \dagger}$ \\
\hline $6 \mathrm{cpds}$ & 0.808 & 0.061 & $0.001^{\dagger \dagger}$ \\
\hline $12 \mathrm{cpds}$ & 0.832 & 0.064 & $0.001^{\dagger \dagger}$ \\
\hline $18 \mathrm{cpds}$ & 0.857 & 0.055 & $0.001^{\dagger \dagger}$ \\
\hline \multicolumn{4}{|l|}{ Rue 28-hue } \\
\hline Number of errors & 0.610 & 0.081 & 0.186 \\
\hline Tritan unspecific errors & 0.714 & 0.074 & $0.01^{\dagger}$ \\
\hline \multicolumn{4}{|l|}{ PDT (sheet) } \\
\hline Successful number of sheets & 0.758 & 0.068 & $0.002^{\dagger \dagger}$ \\
\hline
\end{tabular}

TABle 4: Pearson's correlation between psychophysical tests and Mini Mental State Examination.

\begin{tabular}{lcc}
\hline Psychophysical test & $r$ & $P$ value \\
\hline Visual acuity & 0.457 & $0.001^{\dagger \dagger}$ \\
Contrast sensitivity & & \\
$\quad 3$ cpds & 0.366 & $0.011^{\dagger \dagger}$ \\
6 cpds & 0.516 & $0.001^{\dagger \dagger}$ \\
12 cpds & 0.451 & $0.001^{\dagger \dagger}$ \\
18 cpds & 0.468 & $0.001^{\dagger \dagger}$ \\
Rue 28-hue & & \\
$\quad$ Number of errors & -0.271 & 0.063 \\
$\quad$ Tritan unspecific errors & -0.329 & $0.023^{\dagger}$ \\
PDT & & \\
$\quad$ Successful number of sheets & 0.536 & $0.001^{\dagger \dagger}$ \\
\hline${ }^{\dagger} P<0.05,{ }^{\dagger \dagger} P<0.01$ Pearson's test (AD: Alzheimer's disease; cpds: cycles \\
per degree; PDT: perception digital test).
\end{tabular}

perception. Thus, in MAD patients the spatial frequency of $18 \mathrm{cpds}$ showed the greatest decrease in CS (44.57\%) compared with the age-matched control group. The analysis of the ROC curves (Table 3; Figure 2(b)) showed that, for MAD patients, the highest spatial frequencies analyzed (18 and $12 \mathrm{cpds}$ ) had the widest areas under the ROC curves for all the parameters analyzed. The 18-cpd frequency had the strongest correlation, followed by the 12-cpd frequency. A good correlation was detected also for the 6- and 3-cpd frequencies (Table 3; Figure 2(b)).

A positive and statistically significant linear association was found between CS for all spatial frequencies tested and MMSE score (Table 4).

3.3. Color Perception. The analysis of color vision with the Rue 28-hue test showed that there were no significant differences in the color perception between patients with MAD and age-matched control group based on the total error score (Table 2). However, the analysis of the tritan axis revealed that (i) the number of tritan unspecific errors was significantly increased in MAD patients in comparison with age-matched control (Table 2; Figure 1(c)), (ii) aROC curve drawn for the total tritan unspecific errors was statistically significant (Table 3; Figure 2(c)), and (iii) there was a negative and statistically significant linear association between tritan unspecific errors and the MMSE score (Table 4).

3.4. Perception Digital Test (PDT). The PDT mean value found for the control group was significantly higher than that of the MAD group. The analysis of the individual sheets revealed a significant difference in sheets 2,8 , and 10 between MAD and age-matched control subjects (Table 2; Figure 1(d)).

The aROC curve for PDT showed a statistically significant wide area. However, when the analysis was calculated by individual sheets, no significant differences were found (Table 3; Figure 2(d)). A positive and statistically significant linear association was found between the PDT and MMSE score (Table 4).

\section{Discussion}

AD patients reportedly manifest subjective visual complaints, including the inability to read, spatial deficits, or difficulty in recognizing faces despite having relatively good visual acuity values and visual fields. For this reason the patients in our study were carefully selected so that sample was quite uniform regarding VA and MMSE. In addition, between different SC tests available, the CSV-1000 was chosen because results are more independent with respect to the VA [37].

One of the relevant points of the present study concerns MMSE value. Our MAD patients were homogeneous in terms of their disease stage and had a high MMSE mean value $(23.3 \pm 3.1)$, higher than any value found in the literature $[36,37,49-51]$. This means that all MAD patients included in this study were in a very early stage of the disease.

4.1. Visual Acuity. VA values in the present study were within normal limits for this age range. However, mean VA in MAD patients was significantly decreased in comparison with agematched control subjects. VA values in $\mathrm{AD}$ patients have been controversial. Thus, several studies reported no decrease in VA in patients with Alzheimer's neurodegeneration [36, $40,41,50,52-54]$ while others showed a decrease in VA [55] which in some instances was associated with visual hallucinations [56].

4.2. Contrast Sensitivity. We found that, in comparison with age-matched control subjects, CS in MAD patients was significantly reduced in all spatial frequencies, the reduction being more pronounced at higher frequencies. Specifically, at a spatial frequency of $18 \mathrm{cpds}$, MAD patients showed a $44.57 \%$ CS reduction in comparison with age-matched control. High spatial frequencies are recorded in the P cells of the retina, which are more concentrated in the macular area. Low spatial frequencies are recorded by the $\mathrm{M}$ cells located over the entire retina [57]. Our CS results suggest that MAD patients undergo an impairment of both P- and $\mathrm{M}$-cell function, $\mathrm{P}$ cells being the most affected. This finding 

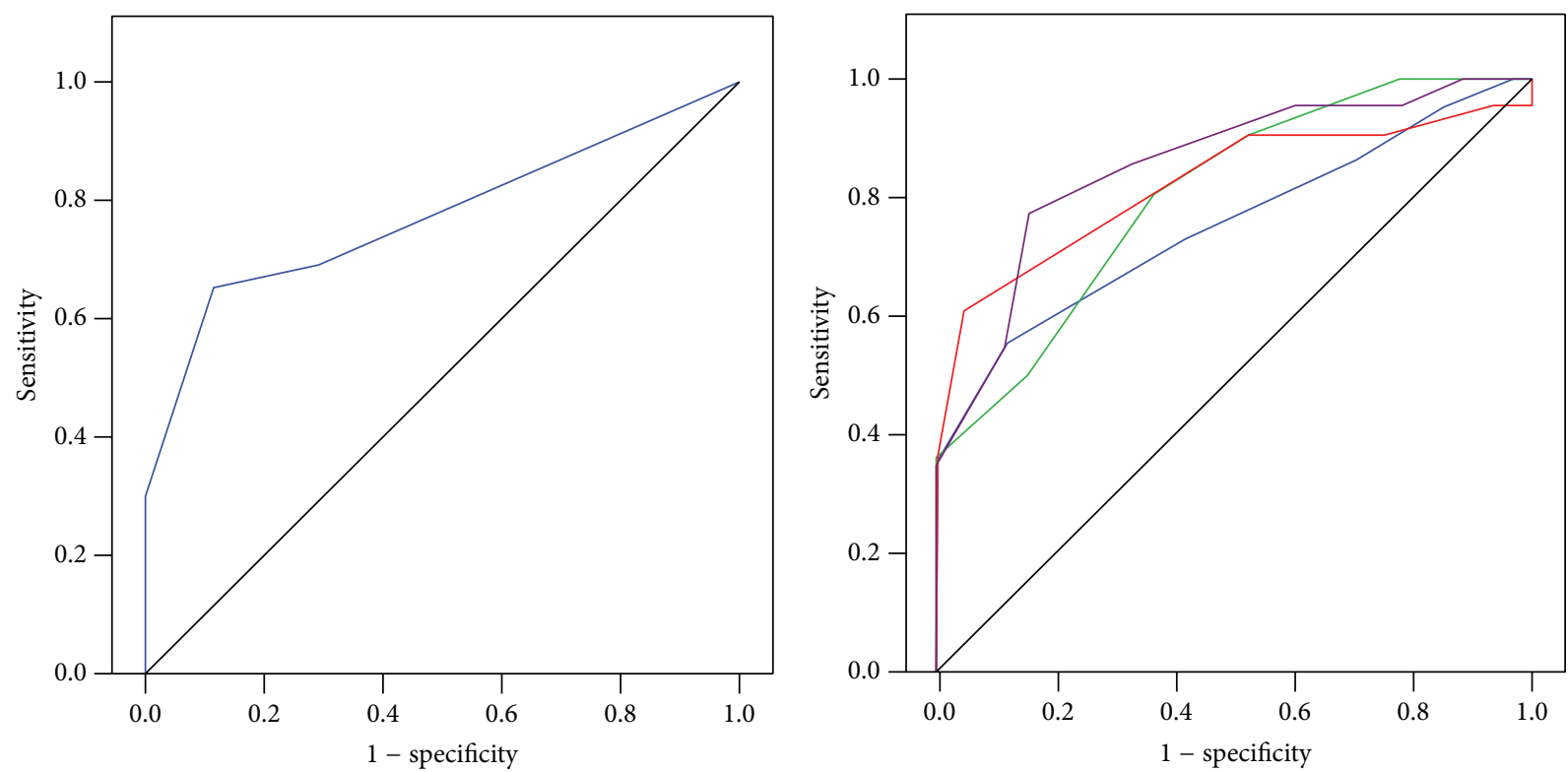

Source of the curve

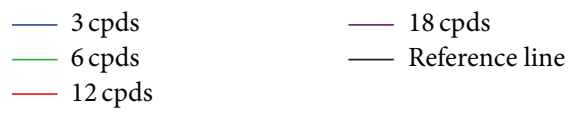

(a) Visual acuity

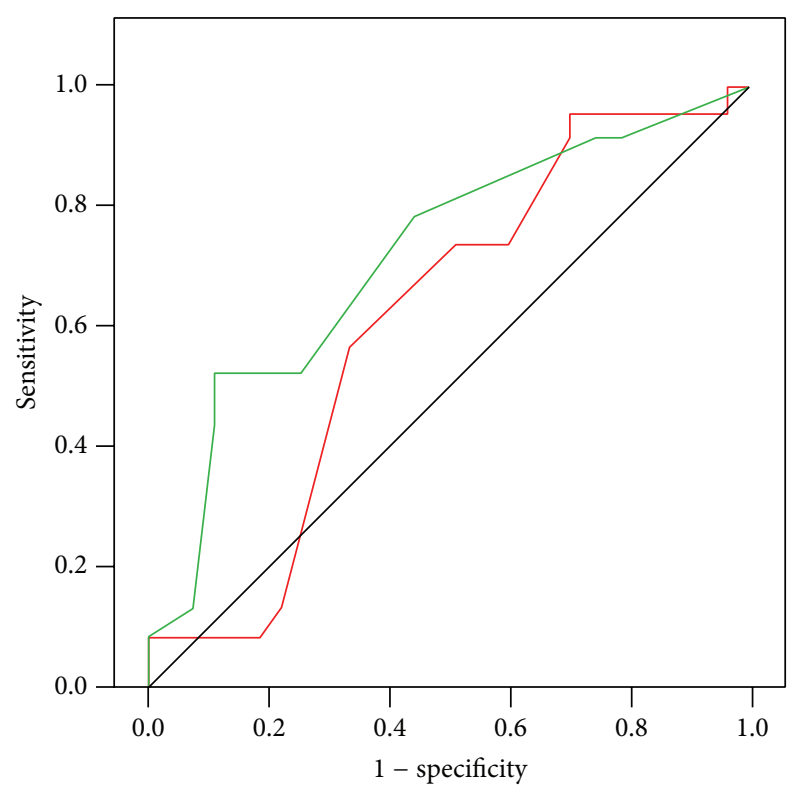

(b) Contrast sensitivity

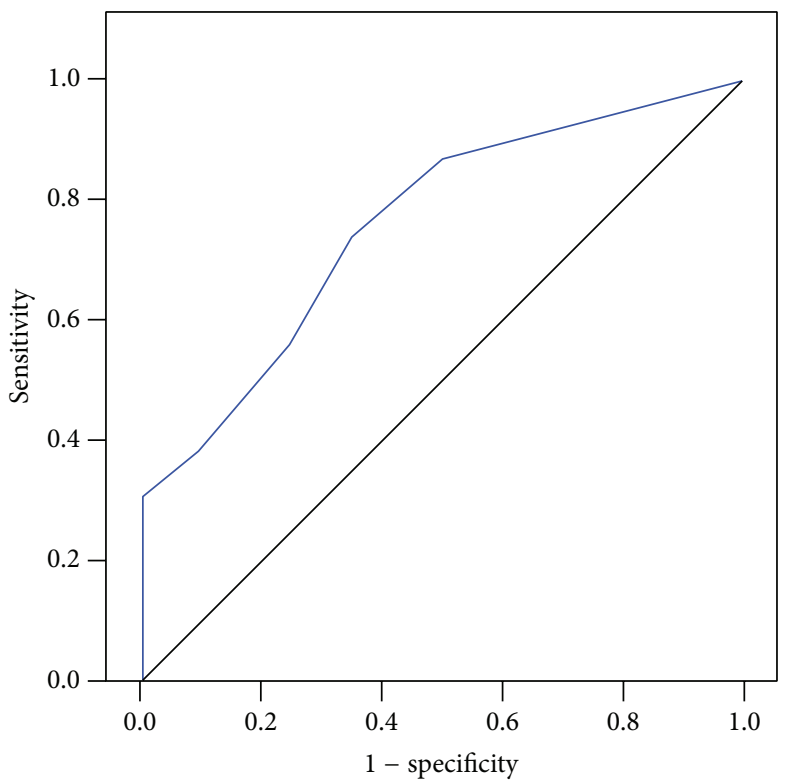

Source of the curve

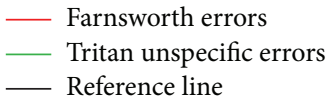

(c) Color vision

(d) Perception digital test

FIGURE 2: Areas under the ROC curves of the psychophysical tests in discriminating between mild AD patients and control subjects. (a) Visual acuity (dec), (b) contrast sensitivity, (c) Rue 28-hue color test, and (d) perception digital test.

correlates with data recently published by our group [9] in which patients with MAD had a significant decrease in the nerve fiber layer thickness in the macular area compared with age-matched controls. By contrast, the significant decrease in CS for the lower spatial frequencies found here in MAD patients did not parallel OCT findings in the peripapillary region in the fact that the decrease in the nerve fiber layer thickness reported by our group in this retinal region did 
not reach significance when compared with controls. These findings tempt us to postulate that, in MAD, CS seems to detect the M pathway impairment earlier than OCT does.

$\mathrm{CS}$ in $\mathrm{AD}$ has been analyzed for several years. Although in two studies no differences between $\mathrm{AD}$ and age-matched control were detected $[40,54]$, most reports found that CS function is impaired in $\mathrm{AD}$ patients. In some instances, CS was reduced at all spatial frequencies examined [34-36, 51, 53, $55,58-60]$, while in others the greater decline corresponded to high spatial frequencies $[34,51,61]$ and in still others the lower spatial frequencies were the most affected $[49,50$, $52,62]$. Possible reasons for such discrepancies may lie in differences among samples and the CS test used [37]. Thus, the Regan chart, a low-contrast letter, and the Vistech VCTS 6500 are influenced by the VA value while the Pelli-Robson test and the Freiburg test are independent of VA [37]. In the present study, we use the CSV-1000 test which is based on the Pelli-Robson test and it can thus be assumed that it is not influenced by VA values.

In agreement with most studies on $\mathrm{CS}$ in $\mathrm{AD}$, CS function was impaired in our cohort of early AD. However, the degree of involvement varied among spatial frequencies, the reduction being greater at the higher ones. Notably, in the studies reported in which CS was equally decreased in all spatial frequencies, $\mathrm{AD}$ patients were at a more advanced stage of the disease than were our MAD patients, as indicated by their lower MMSE values. There are only two studies with results consistent with ours, showing an increased decline at the higher spatial frequencies. However, both studies presented methodological differences with respect to ours. In the Neargarder et al. study [37], the results could have been influenced by the poorer VA of the patients, the test selected, and the great variability of MMSE (6-26) in the AD group. Meanwhile, the Gilmore et al. study [63] included only the participants who provided a valid response across all spatial frequencies, removing any patients who were not able to discriminate all checked spatial frequencies. Given these data, it seems that the 12- and 18-cpd spatial frequencies are the most affected in the early stages on the disease and thus as the disease progresses, all spatial frequencies become equally involved. According to our ROC values, the most sensitive spatial frequencies were $18 \mathrm{cpds}$ (the highest), followed by the 12 cpds ( $85.7 \%$ and $83.2 \%$, resp.); therefore, the highest spatial frequencies appear to have the highest diagnostic value in MAD neurodegeneration.

The fact that CS is impaired in AD patients in comparison with healthy elderly subjects has significant implications for cognitive abilities and daily function of $\mathrm{AD}$ patients [37], especially when the first spatial frequencies affected, according to our data, appear to be those corresponding to macular function.

4.3. Color Perception. When assessing color perception in our MAD patients, we found no significant differences in terms of color defects in comparison with age-matched control subjects. However, we found that our MAD patients made significantly more $(72.62 \%)$ unspecific errors in the tritan (blue) axis than did the controls, which represented almost a threefold increase in the errors committed by the MAD group.

Unlike subjects with a loss of color vision due to focal cortical lesions, patients with $\mathrm{AD}$ rarely complain of colorvision deficits. Color testing in patients with $\mathrm{AD}$ is controversial because these patients have a deficit in naming colors and therefore might have trouble verbalizing the colors or nominating numbers and shapes that are being viewed. The color test used in the present study does not require naming, although it should be taken into account that test performance depends on the memory of the patients and therefore, for appropriate testing, the instructions need to be repeated by the examiner during test session, when necessary.

Some studies using the Farnsworth and Ishihara tests have found no color perception differences between patients with $\mathrm{AD}$ and the control group [58,64,65]. On the other hand, several studies using the City University Color Test found defects in the tritan axis and reported a correlation with the degree of dementia $[30,50,66]$. These data are consistent with the results reported by other authors using the Ishihara test [55] and the City University Color Test [67, 68]. By contrast, Pache et al., using the Ishihara test and the PV-16, found that unspecific errors not associated with a specific axis were more prevalent in $\mathrm{AD}$ patients compared to controls; however, this finding was not related to the severity of the disease [31]. This discrepancy among color-vision results in $\mathrm{AD}$ patients may be due to differences in the color test applied, so that comparisons of the results are difficult to interpret.

Salamone et al. postulated that the problem of color discrimination in patients with $\mathrm{AD}$ is not purely cognitive but seems to be related to damage in the structures responsible for the perception of color stimuli [32]. This statement is consistent with the evidence that extrastriate lesions may result in tritanomalous color deficits [50] and that the extrastriate cortex is severely affected in AD. The fact that no tritanomaly was found in our MAD patients but an increment of unspecific errors appeared along the tritan axis leads us to postulate that, in addition to the general loss of $\mathrm{M}$ cells and $\mathrm{P}$ cells that seems to be taking place in our patients (as mentioned above), the $\mathrm{K}$ pathway may also be involved, given that the blue-yellow spectrum is associated with this pathway [69]. Bistratified ganglion cells receive blue-on/yellow-off color-opponent excitation signals from the short-wavelength sensitive $S$ cones and project this information to the koniocellular layers in the LGN [70].

4.4. Perception Digital Test (PDT). The MAD patients of the present study made significantly more mistakes in PDT answers than did the age-matched control subjects. Given that no statistically significant differences in educational level were found among groups, the influence of this variable could be ruled out.

The part of the brain initially involved in AD is the temporal cortex, which is then followed by the temporoparietal association cortex [1, 71]. As a consequence, visual perception disorders are frequently associated with Alzheimer's neurodegeneration [71]. Before PDT development [46] visuoperceptual tests showed low sensitivity for detecting disorders in 
the early stages of the disease [72]. The development of more sensitive neuropsychological tests such as PDT has enabled the assessment of visual perception disorders in initial $\mathrm{AD}$ [46]. In view of the good predictive value $(\mathrm{aROC}=0.758, P<$ 0.01 ) provided by the application of the PDT, we postulate PDT as a useful ancillary screening test for MAD.

4.5. aROC. To the best of our knowledge, no predictive (ROC) analysis is available for the diagnosis value of different visual psychophysical test in $\mathrm{AD}$. A noteworthy finding of the present work was that the test with the greatest prognostic value in MAD patients was CS for 18-cpd and 12-cpd spatial frequencies (aROC 0.857 and 0.832 , resp.), followed by VA (aROC 0.771), PDT (aROC 0.758), and unspecific errors in the blue region (aROC 0.714).

4.6. Pearson's Correlation. As mentioned above, our $\mathrm{AD}$ patients had a high mean MMSE value, the highest so far reported to the best of our knowledge. However, given that MMSE values ranged from 17 to 31, we investigated whether the MMSE value influences the test outcome. As a result, we found a significant correlation between MMSE values and PDT $(r=0.536)$, followed by the CS for 6 -cpd $(r=0.516)$ and 18-cpd $(r=0.468)$ spatial frequencies, and VA $(r=$ $0.457)$ and CS for 12-cpd $(r=0.451)$ and 3-cpd $(r=0.366)$ spatial frequencies. A negative correlation was also found between MMSE and the unspecific errors in the tritan region $(r=-0329)$. All these findings showed that as the disease progresses, the answers to different tests used for assessing visual skills worsened.

Possible drawbacks of the study include the crosssectional design, the subjective nature of the psychophysical testing (which depends on cognitive abilities), and the early stage of disease (mild cognitive impairment, which can be due to reasons other than $\mathrm{AD}$ ). Another limitation could be the sample size. Gathering large samples is a difficult task when dealing with MAD patients. This was especially true in the present study in which the inclusion criteria were very demanding in order to recruit patients in early stages of $\mathrm{AD}$ who were also free of ocular pathology, the latter requisite limiting patient enrollment given the age range of the sample. Nevertheless, our sample size was comparable to that reported in the literature $[36,37,40,50,51,59]$.

\section{Conclusions}

In conclusion, our study demonstrates a deficit in visual perception in early stages of $\mathrm{AD}$, manifested by reductions in VA, CS, and color discrimination. These psychophysical changes correlate with retinal morphological changes (decreased RNFL macular thickness as detected by OCT) detected in the same group of patients [9]. Furthermore, the observed changes in PDT suggest a visual integration deficit, possibly due to, at least in part, an incipient cortical dysfunction. The study of the predictive value of the tests analyzed herein showed that, in the most incipient $\mathrm{AD}$ grades, tests having the greatest predictive value are the CS, VA, unspecific errors in tritan region, and the PDT. The fact that no differences were detected between our MAD patients and age-matched control subjects in the dilated fundus examination highlights the importance of applying psychophysical tests in patients with MAD. Given the difficulty in gathering larger samples in $\mathrm{AD}$, broader transverse as well as longitudinal studies would be useful to track changes in the psychophysical tests as the disease progresses.

\section{Conflict of Interests}

The authors report no conflict of interests and have no proprietary interest in any of the materials mentioned in this paper.

\section{Acknowledgments}

This work was supported by RETICs prevención, detección precoz y tratamiento de la patología ocular prevalente degenerativa y crónica (OFTARED) (Grant ISCIII RD12/ 0034/0002, Spanish Ministry of Science and Innovation); grants from Elena Salobrar-Garcia are currently supported by a Predoctoral Fellowship (FPU) from the Spanish Ministry of Education, Culture and Sport. David Nesbitt corrected the English version of this work.

\section{References}

[1] G. D. Rabinovici, W. W. Seeley, E. J. Kim et al., "Distinct MRI atrophy patterns in autopsy-proven Alzheimer's disease and frontotemporal lobar degeneration," The American Journal of Alzheimer's Disease \& Other Dementias, vol. 22, no. 6, pp. 474488, 2007-2008.

[2] A. London, I. Benhar, and M. Schwartz, "The retina as a window to the brain-from eye research to CNS disorders," Nature Reviews Neurology, vol. 9, no. 1, pp. 44-53, 2013.

[3] N. Patton, T. Aslam, T. MacGillivray, A. Pattie, I. J. Deary, and B. Dhillon, "Retinal vascular image analysis as a potential screening tool for cerebrovascular disease: a rationale based on homology between cerebral and retinal microvasculatures," Journal of Anatomy, vol. 206, no. 4, pp. 319-348, 2005.

[4] M. H. Dehabadi, B. M. Davis, T. K. Wong, and M. F. Cordeiro, "Retinal manifestations of Alzheimer's disease," Neurodegenerative Disease Management, vol. 4, no. 3, pp. 241-252, 2014.

[5] D. R. Hinton, A. A. Sadun, J. C. Blanks, and C. A. Miller, "Opticnerve degeneration in Alzheimer's disease," The New England Journal of Medicine, vol. 315, no. 8, pp. 485-487, 1986.

[6] J. C. Blanks, D. R. Hinton, A. A. Sadun, and C. A. Miller, "Retinal ganglion cell degeneration in Alzheimer's disease," Brain Research, vol. 501, no. 2, pp. 364-372, 1989.

[7] J. C. Blanks, S. Y. Schmidt, Y. Torigoe, K. V. Porrello, D. R. Hinton, and R. H. I. Blanks, "Retinal pathology in Alzheimer's disease. II. Regional neuron loss and glial changes in GCL," Neurobiology of Aging, vol. 17, no. 3, pp. 385-395, 1996.

[8] J. C. Blanks, Y. Torigoe, D. R. Hinton, and R. H. I. Blanks, "Retinal pathology in Alzheimer's disease. I. Ganglion cell loss in foveal/parafoveal retina," Neurobiology of Aging, vol. 17, no. 3, pp. 377-384, 1996.

[9] E. S. Garcia-Martin, B. Rojas, A. I. Ramirez et al., "Macular thickness as a potential biomarker of mild Alzheimer's disease," Ophthalmology, vol. 121, no. 5, pp. 1149-1151, 2014. 
[10] P. K. Iseri, Ö. Altinaş, T. Tokay, and N. Yüksel, "Relationship between cognitive impairment and retinal morphological and visual functional abnormalities in Alzheimer disease," Journal of Neuro-Ophthalmology, vol. 26, no. 1, pp. 18-24, 2006.

[11] V. Parisi, R. Restuccia, F. Fattapposta, C. Mina, M. G. Bucci, and F. Pierelli, "Morphological and functional retinal impairment in Alzheimer's disease patients," Clinical Neurophysiology, vol. 112, no. 10, pp. 1860-1867, 2001.

[12] C. Paquet, M. Boissonnot, F. Roger, P. Dighiero, R. Gil, and J. Hugon, "Abnormal retinal thickness in patients with mild cognitive impairment and Alzheimer's disease," Neuroscience Letters, vol. 420, no. 2, pp. 97-99, 2007.

[13] Y. Lu, Z. Li, X. Zhang et al., "Retinal nerve fiber layer structure abnormalities in early Alzheimer's disease: evidence in optical coherence tomography," Neuroscience Letters, vol. 480, no. 1, pp. 69-72, 2010.

[14] F. Berisha, G. T. Feke, C. L. Trempe, J. W. McMeel, and C. L. Schepens, "Retinal abnormalities in early Alzheimer's disease," Investigative Ophthalmology and Visual Science, vol. 48, no. 5, pp. 2285-2289, 2007.

[15] R. Kromer, N. Serbecic, L. Hausner, F. Aboul-Enein, L. Froelich, and S. C. Beutelspacher, "Detection of retinal nerve fiber layer defects in Alzheimer's disease using SD-OCT,' Frontiers in Psychiatry, vol. 5, article 22, 2014.

[16] A. Kesler, V. Vakhapova, A. D. Korczyn, E. Naftaliev, and M. Neudorfer, "Retinal thickness in patients with mild cognitive impairment and Alzheimer's disease," Clinical Neurology and Neurosurgery, vol. 113, no. 7, pp. 523-526, 2011.

[17] C. Y. Cheung, Y. T. Ong, S. Hilal et al., "Retinal ganglion cell analysis using high-definition optical coherence tomography in patients with mild cognitive impairment and Alzheimer's disease," Journal of Alzheimer's Disease, vol. 45, no. 1, pp. 45-56, 2015.

[18] E. O. Oktem, E. Derle, S. Kibaroglu, C. Oktem, I. Akkoyun, and U. Can, "The relationship between the degree of cognitive impairment and retinal nerve fiber layer thickness," Neurological Sciences, 2015.

[19] D. Liu, L. Zhang, Z. Li et al., "Thinner changes of the retinal nerve fiber layer in patients with mild cognitive impairment and Alzheimer's disease," BMC Neurology, vol. 15, article 14, 2015.

[20] M. Koronyo-Hamaoui, Y. Koronyo, A. V. Ljubimov et al., "Identification of amyloid plaques in retinas from Alzheimer's patients and noninvasive in vivo optical imaging of retinal plaques in a mouse model," NeuroImage, vol. 54, no. 1, pp. S204S217, 2011.

[21] Y. Tsai, B. Lu, A. V. Ljubimov et al., "Ocular changes in TGF344-AD rat model of Alzheimer's disease," Investigative Ophthalmology and Visual Science, vol. 55, no. 1, pp. 523-534, 2014.

[22] B. Liu, S. Rasool, Z. Yang et al., "Amyloid-peptide vaccinations reduce $\beta$-amyloid plaques but exacerbate vascular deposition and inflammation in the retina of Alzheimer's transgenic mice," The American Journal of Pathology, vol. 175, no. 5, pp. 2099-2110, 2009.

[23] U. Kayabasi, R. Sergott, and M. Rispoli, "Retinal examination for the diagnosis of Alzheimer's disease," Journal of Clinical Ophthalmology and Research, vol. 3, article 4, 2014.

[24] M. C. Campbell, D. DeVries, L. Emptage et al., "Polarization properties of amyloid beta in the retina of the eye as a biomarker of Alzheimer's disease," in Bio-Optics: Design and Application, p. BM3A.4, OSA Publishing, 2015.
[25] Y. Koronyo, B. C. Salumbides, K. L. Black, and M. KoronyoHamaoui, "Alzheimer's disease in the retina: Imaging retinal a $\beta$ plaques for early diagnosis and therapy assessment," Neurodegenerative Diseases, vol. 10, no. 1-4, pp. 285-293, 2012.

[26] P. R. Hof and J. H. Morrison, "Quantitative analysis of a vulnerable subset of pyramidal neurons in Alzheimer's disease: II. Primary and secondary visual cortex," Journal of Comparative Neurology, vol. 301, no. 1, pp. 55-64, 1990.

[27] G. L. Trick, L. R. Trick, P. Morris, and M. Wolf, "Visual field loss in senile dementia of the Alzheimer's type," Neurology, vol. 45, no. 1, pp. 68-74, 1995.

[28] R. Steffes and J. Thralow, "Visual field limitation in the patient with dementia of the Alzheimer's type," Journal of the American Geriatrics Society, vol. 35, no. 3, pp. 198-204, 1987.

[29] S. L. Risacher, D. WuDunn, S. M. Pepin et al., "Visual contrast sensitivity in Alzheimer's disease, mild cognitive impairment, and older adults with cognitive complaints," Neurobiology of Aging, vol. 34, no. 4, pp. 1133-1144, 2013.

[30] A. Cronin-Golomb, R. Sugiura, S. Corkin, and J. H. Growdon, "Incomplete Achromatopsia in Alzheimer's disease," Neurobiology of Aging, vol. 14, no. 5, pp. 471-477, 1993.

[31] M. Pache, C. H. W. Smeets, P. F. Gasio et al., "Colour vision deficiencies in Alzheimer's disease," Age and Ageing, vol. 32, no. 4, pp. 422-426, 2003.

[32] G. Salamone, C. Di Lorenzo, S. Mosti et al., "Color discrimination performance in patients with Alzheimer's disease," Dementia and Geriatric Cognitive Disorders, vol. 27, no. 6, pp. 501-507, 2009.

[33] K. Krasodomska, W. Lubiński, A. Potemkowski, and K. Honczarenko, "Pattern electroretinogram (PERG) and pattern visual evoked potential (PVEP) in the early stages of Alzheimer's disease," Documenta Ophthalmologica, vol. 121, no. 2, pp. 111-121, 2010.

[34] G. L. Trick, M. C. Barris, and M. Bickler-Bluth, "Abnormal pattern electroretinograms in patients with senile dementia of the Alzheimer type," Annals of Neurology, vol. 26, no. 2, pp. 226231, 1989.

[35] A. Cronin-Golomb, J. F. Rizzo, S. Corkin, and J. H. Growdon, "Visual function in Alzheimer's disease and normal aging," Annals of the New York Academy of Sciences, vol. 640, pp. 2835, 1991.

[36] V. Lakshminarayanan, J. Lagrave, M. L. Kean, M. Dick, and R. Shankle, "Vision in dementia: contrast effects," Neurological Research, vol. 18, no. 1, pp. 9-15, 1996.

[37] S. A. Neargarder, E. R. Stone, A. Cronin-Golomb, and S. Oross, "The impact of acuity on performance of four clinical measures of contrast sensitivity in Alzheimer's disease," Journals of Gerontology, Series B: Psychological Sciences and Social Sciences, vol. 58, no. 1, pp. P54-P62, 2003.

[38] D. A. Valenti, "Alzheimer's disease: visual system review," Optometry, vol. 81, no. 1, pp. 12-21, 2010.

[39] I. B. Wollner and S. P. Diamond, "The measurement of spatial contrast sensitivity in cases of blurred vision associated with cerebral lesions," Brain, vol. 99, no. 4, pp. 695-710, 1976.

[40] G. Schlotterer, M. Moscovitch, and D. Crapper-McLachlan, "Visual processing deficits as assessed by spatial frequency contrast sensitivity and backward masking in normal ageing and Alzheimer's disease," Brain, vol. 107, no. 1, pp.309-324, 1984.

[41] C. E. Wright, N. Drasdo, and G. F. A. Harding, "Pathology of the optic nerve and visual association areas. Information given by the flash and pattern visual evoked potential, and the temporal 
and spatial contrast sensitivity function," Brain, vol. 110, no. 1, pp. 107-120, 1987.

[42] A. Cronin-Golomb, S. Corkin, and J. H. Growdon, "Contrast sensitivity in Alzheimer's disease," Journal of the Optical Society of America A, vol. 4, p. 7, 1987.

[43] L. Gao, Y. Liu, X. Li, Q. Bai, and P. Liu, "Abnormal retinal nerve fiber layer thickness and macula lutea in patients with mild cognitive impairment and Alzheimer's disease," Archives of Gerontology and Geriatrics, vol. 60, no. 1, pp. 162-167, 2015.

[44] E. Marziani, S. Pomati, P. Ramolfo et al., "Evaluation of retinal nerve fiber layer and ganglion cell layer thickness in Alzheimer's disease using spectral-domain optical coherence tomography," Investigative Ophthalmology \& Visual Science, vol. 54, no. 9, pp. 5953-5958, 2013.

[45] B. Reisberg, S. H. Ferris, M. J. de Leon, and T. Crook, "The global deterioration scale for assessment of primary degenerative dementia," The American Journal of Psychiatry, vol. 139, no. 9, pp. 1136-1139, 1982.

[46] L. Rami, M. Serradell, B. Bosch, A. Villar, and J. L. Molinuevo, "Perception Digital Test (PDT) for the assessment of incipient visual disorder in initial Alzheimer's disease," Neurologia, vol. 22, no. 6, pp. 342-347, 2007.

[47] A. Roth, Test-28 hue de Roth selon Farnsworth-Munsell (Manual), Luneau, Paris, France, 1966.

[48] D. Farnsworth and M. Color, The Farnsworth-Munsell 100-Hue Test for the Examination of Color Discrimination, Munsell Color Company, 1957.

[49] A. Cronin-Golomb, G. C. Gilmore, S. Neargarder, S. R. Morrison, and T. M. Laudate, "Enhanced stimulus strength improves visual cognition in aging and Alzheimer's disease," Cortex, vol. 43, no. 7, pp. 952-966, 2007.

[50] A. Cronin-Golomb, S. Corkin, J. F. Rizzo, J. Cohen, J. H. Growdon, and K. S. Banks, "Visual dysfunction in Alzheimer's disease: relation to normal aging," Annals of Neurology, vol. 29, no. 1, pp. 41-52, 1991.

[51] G. C. Gilmore and P. J. Whitehouse, "Contrast sensitivity in Alzheimer's disease: a 1-year longitudinal analysis," Optometry \& Vision Science, vol. 72, no. 2, pp. 83-91, 1995.

[52] D. N. Levine, J. M. Lee, and C. M. Fisher, "The visual variant of Alzheimer's disease: a clinicopathologic case study," Neurology, vol. 43, no. 2, pp. 305-313, 1993.

[53] M. F. Mendez, R. L. Tomsak, and B. Remler, "Disorders of the visual system in Alzheimer's disease," Journal of Clinical NeuroOphthalmology, vol. 10, no. 1, pp. 62-69, 1990.

[54] M. Rizzo and M. Nawrot, "Perception of movement and shape in Alzheimer's disease,” Brain, vol. 121, no. 12, pp. 2259-2270, 1998.

[55] A. A. Sadun, M. Borchert, E. DeVita, D. R. Hinton, and C. J. Bassi, "Assessment of visual impairment in patients with Alzheimer's disease," The American Journal of Ophthalmology, vol. 104, no. 2, pp. 113-120, 1987.

[56] C. Murgatroyd and R. Prettyman, "An investigation of visual hallucinosis and visual sensory status in dementia," International Journal of Geriatric Psychiatry, vol. 16, no. 7, pp. 709-713, 2001.

[57] M. S. Livingstone and D. H. Hubel, "Psychophysical evidence for separate channels for the perception of form, color, movement, and depth," The Journal of Neuroscience, vol. 7, no. 11, pp. 3416-3468, 1987.

[58] C. J. Bassi, K. Solomon, and D. Young, "Vision in aging and dementia," Optometry \& Vision Science, vol. 70, no. 10, pp. 809813, 1993.
[59] R. W. Crow, L. B. Levin, L. LaBree, R. Rubin, and S. E. Feldon, "Sweep visual evoked potential evaluation of contrast sensitivity in Alzheimer's dementia," Investigative Ophthalmologye Visual Science, vol. 44, no. 2, pp. 875-878, 2003.

[60] F. K. Cormack, M. Tovee, and C. Ballard, "Contrast sensitivity and visual acuity in patients with Alzheimer's disease," International Journal of Geriatric Psychiatry, vol. 15, no. 7, pp. 614-620, 2000.

[61] J. T. Hutton, J. L. Morris, J. W. Elias, and J. N. Poston, “Contrast sensitivity dysfunction in Alzheimer's disease," Neurology, vol. 43, no. 11, pp. 2328-2330, 1993.

[62] D. R. Baker, M. F. Mendez, J. C. Townsend, P. F. Ilsen, and D. C. Bright, "Optometric management of patients with Alzheimer's disease," Journal of the American Optometric Association, vol. 68, no. 8, pp. 483-494, 1997.

[63] G. C. Gilmore, A. Cronin-Golomb, S. A. Neargarder, and S. R. Morrison, "Enhanced stimulus contrast normalizes visual processing of rapidly presented letters in Alzheimer's disease," Vision Research, vol. 45, no. 8, pp. 1013-1020, 2005.

[64] S. Wood, K. F. Mortel, M. Hiscock, B. G. Breitmeyer, and J. S. Caroselli, "Adaptive and maladaptive utilization of color cues by patients with mild to moderate Alzheimer's disease," Archives of Clinical Neuropsychology, vol. 12, no. 5, pp. 483-489, 1997.

[65] F. Massoud, H. Chertkow, V. Whitehead, O. Overbury, and H. Bergman, "Word-reading thresholds in Alzheimer disease and mild memory loss: a pilot study," Alzheimer Disease \& Associated Disorders, vol. 16, no. 1, pp. 31-39, 2002.

[66] A. Cronin-Golomb, S. Corkin, and J. H. Growdon, "Visual dysfunction predicts cognitive deficits in Alzheimer's disease," Optometry \& Vision Science, vol. 72, no. 3, pp. 168-176, 1995.

[67] J. F. Rizzo III, A. Cronin-Golomb, J. H. Growdon et al., "Retinocalcarine function in Alzheimer's disease: a clinical and electrophysiological study," Archives of Neurology, vol. 49, no. 1, pp. 93-101, 1992.

[68] A. Cronin-Golomb, S. Corkin, and J. H. Growdon, "Visual dysfunction predicts cognitive deficits in Alzheimer's disease," Optometry and Vision Science, vol. 72, no. 3, pp. 168-176, 1995.

[69] P. R. Martin, A. J. R. White, A. K. Goodchild, H. D. Wilder, and A. E. Sefton, "Evidence that blue-on cells are part of the third geniculocortical pathway in primates," European Journal of Neuroscience, vol. 9, no. 7, pp. 1536-1541, 1997.

[70] D. M. Dacey and B. B. Lee, “The 'blue-on' opponent pathway in primate retina originates from a distinct bistratified ganglion cell type," Nature, vol. 367, no. 6465, pp. 731-735, 1994.

[71] K. L. Possin, "Visual spatial cognition in neurodegenerative disease," Neurocase, vol. 16, no. 6, pp. 466-487, 2010.

[72] A. L. Benton, Contributions to Neuropsychological Assessment: A Clinical Manual, Oxford University Press, Oxford, UK, 1994. 


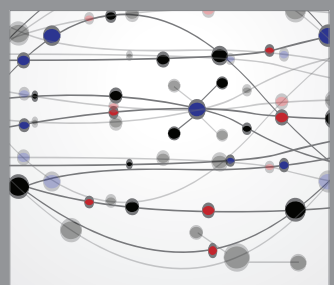

The Scientific World Journal
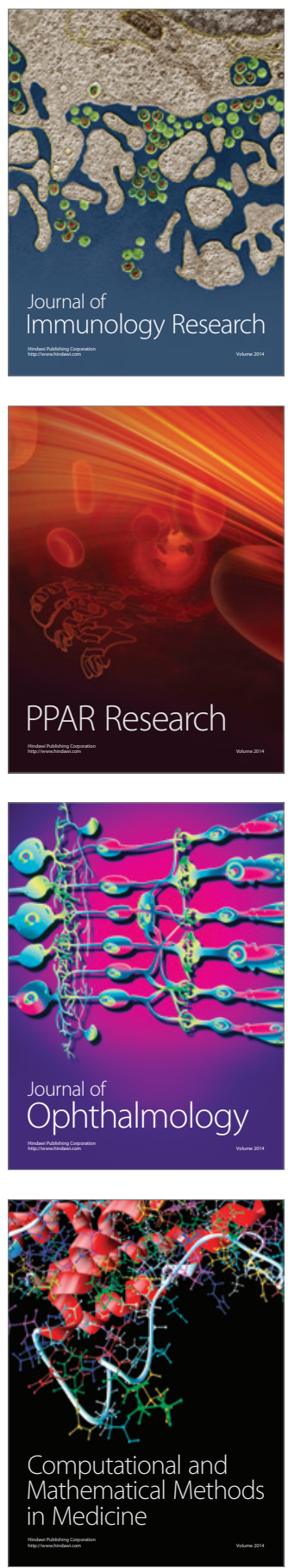

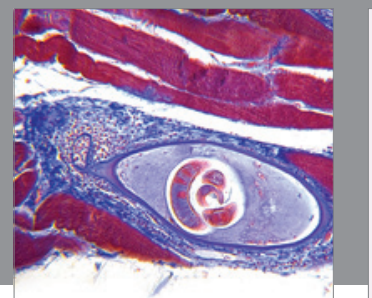

Gastroenterology

Research and Practice
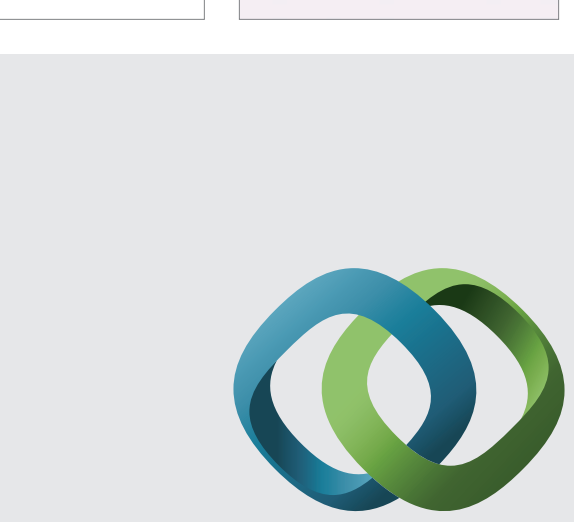

\section{Hindawi}

Submit your manuscripts at

http://www.hindawi.com
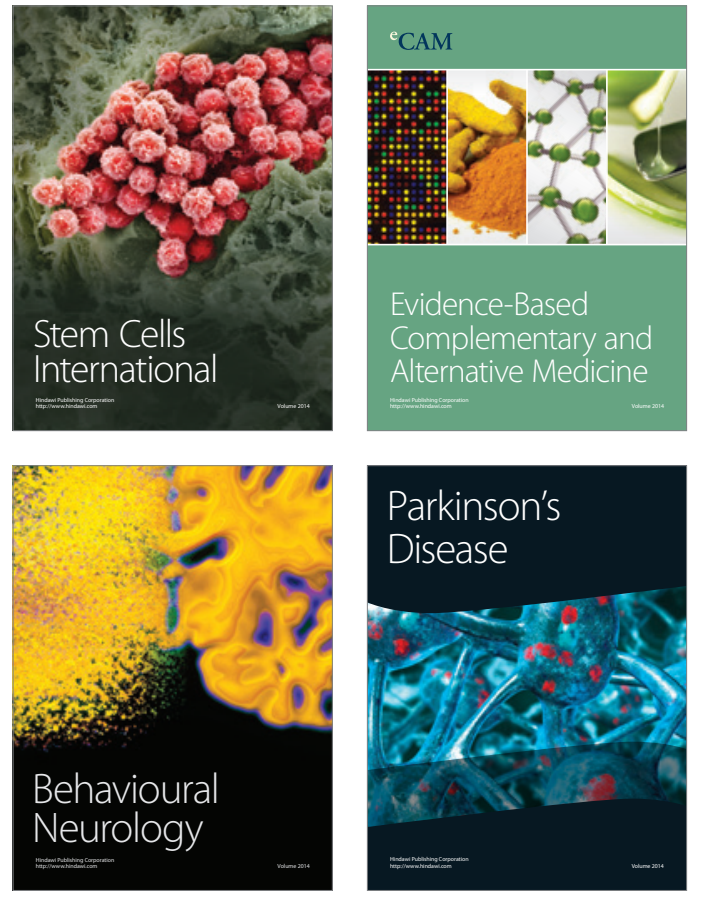
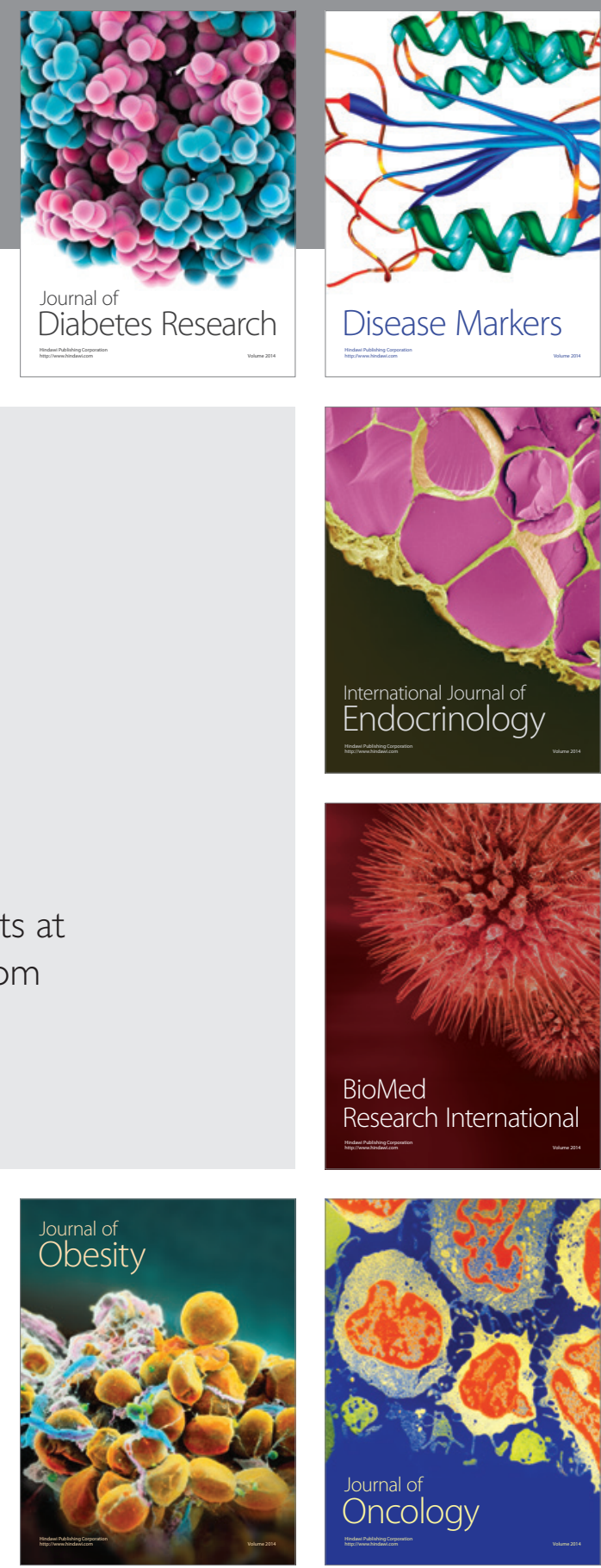

Disease Markers
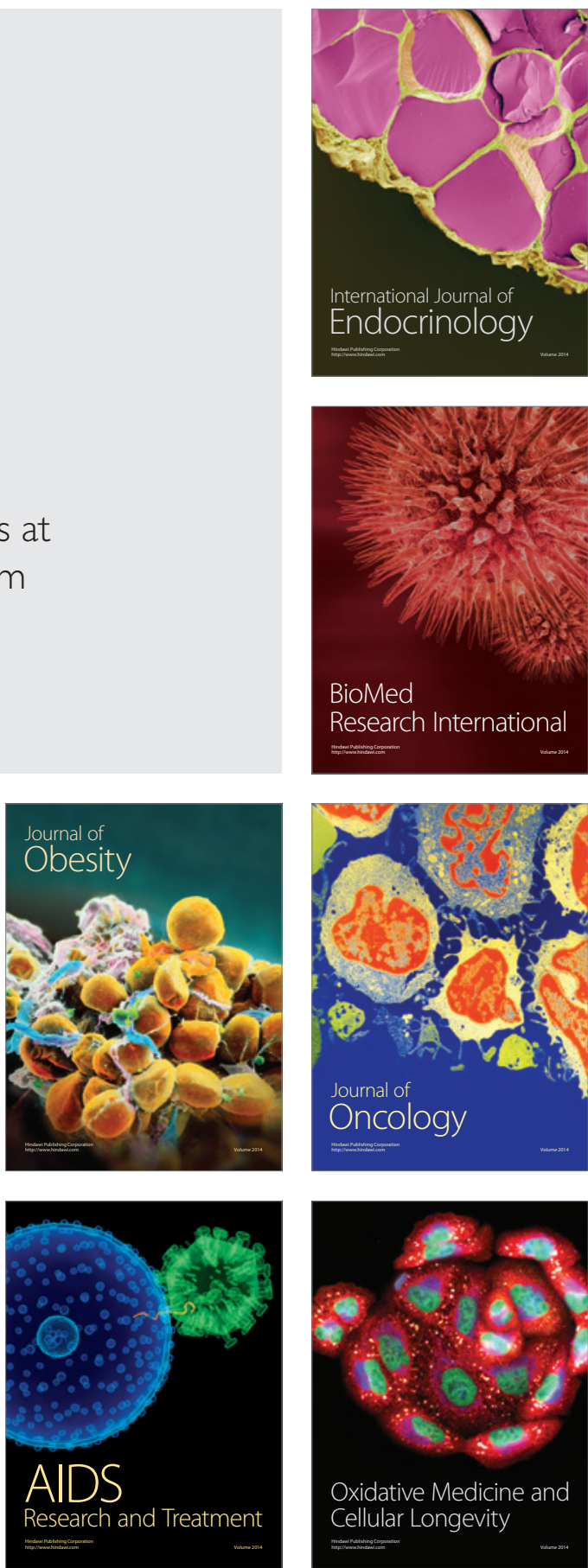